Case Report

\title{
INTRAOCULAR FOREIGN BODY-A PECULIAR CHALLENGE
}

\author{
Keshav K Bhat ${ }^{1}$, Chinnappa A G ${ }^{2}$, M uralee Mohan ${ }^{3}$, Harish Shetty \& Suman Banerjee \\ ${ }^{1}$ Consultant M axillofacial Surgeon, The Face Clinic, Mangalore , ${ }^{2}$ Consultant Ophthalmologist, Prasad Nethralaya, Udupi, \\ ${ }^{3}$ Professor, ${ }^{5}$ Post Graduate, Department of Oral \& M axillofacial Surgery, A.B. Shetty M emorial Institute of Dental Sciences, \\ ${ }^{4}$ Professor, Department of Opthalmology, K.S. Hegde Medical Academy, Nitte University, M angalore, Karnataka, India \\ Correspondence : \\ Muralee Mohan \\ Professor, Department of Oral \& M axillofacial Surgery, A.B. Shetty M emorial Institute of Dental Sciences, \\ Nitte University, Mangalore - 575 018. Karnataka, India \\ E-mail : drmuraleemohan@gmail.com
}

\begin{abstract}
:
Intraorbital foreign bodies often present a confusing clinical picture and managing them remains a challenging experience to the oral and maxillofacial surgeons. Wooden foreign bodies are notorious for remaining quiescent for a long time, before presenting with a variety of complications. The wound of entry may often be small and self-sealing. Wooden foreign bodies also show a propensity to break during attempted removal. Intraorbital wood is often not detected by standard diagnostic tests like the computed tomography scan, adding to the diagnostic dilemma. A retained foreign body can give rise to serious complications, the most devastating of which is loss of the eye. This interventional case report of an unusual case of a wooden intraorbital foreign body reviews the clinical features, radiological appearance and surgical management. Details of ocular history, preoperative ocular examination findings including visual acuity, computed tomography findings were noted. Early surgical exploration was carried out with blunt dissection and careful hemostasis. Thus the foreign body extraction greatly influenced the visual prognosis and final outcome of the patient.
\end{abstract}

Keywords: intraorbital, wooden foreign bodies, surgical removal.

\section{Introduction :}

Management of foreign bodies especially in the eye is a challenging experience for most of the maxillofacial surgeons. Presence of foreign body should be suspected if the patient presents with severe infection or a sensation of foreign body within the orbit. Corneal abrasions may be present even after the removal of foreign body.

Intraocular foreign bodies can be categorized into superficial and penetrating foreign bodies. Superficial foreign bodies are those that stick to the front of the eye or get trapped under one of the eyelids, but do not enter the eye. Penetrating foreign bodies are those penetrate the outer layer of the eye (cornea or sclera) and enter the eye.

\begin{tabular}{|c|}
\hline Access this article online \\
\hline Quick Response Code \\
\hline
\end{tabular}

Appropriate diagnostic imaging technique such as computed tomography is necessary before planning the removal of intraocular foreign bodies.

The time and method of removal of foreign bodies of the orbit depend on various factors like size, shape, nature ,size of impingement ,potential for developing any complications like optic nerve compression, infections etc. Organic foreign bodies like wood have to be removed as soon as possible due to associated risk of infections. It has been seen that foreign bodies of greater mass are associated with worse visual outcome and warrants early removal.

A peculiar case-report of intraocular foreign body is presented in this article.

\section{Case Report :}

A 39 year old female patient reported to us with history of penetration of thin wooden stick into her right eye at her workplace 2 days back. She also complained of dull aching pain, swelling of right eye, excessive tears from the same eye, burning sensation and reduced mouth opening.

On clinical examination, the patient was well oriented to time, place and person. A $0.5 \times 0.5 \mathrm{~cm}$ laceration was noticed over the right conjunctiva. About $1 \mathrm{~cm}$ broom splinter was seen protruding out from the lateral aspect of 
right eye and penetrating the sclera. The eye was inflamed, tender and edematous with slight subconjunctival hemorrhage. However pupils were equal in size with normal light reflex. She had no diplopia and her eye movements were normal in all gazes. Her mouth opening was about $20 \mathrm{~mm}$. On palpation right lateral perygoid muscle was found to be tender.

Ophthalmic consultation was done for the same patient. The ophthalmologic report confirmed the presence of foreign body in the right eye. However diplopia or blindness was not reported. The visual acuity was $6 / 6$ according to Snell's chart. Eye movements were normal in all gazes. Intraocular pressure in the affected eye was within normal limits.

Plain sagittal CT of the orbit showed hypodense linear foreign body passing through lateral wall of right orbit through the right maxillary sinus.

The patient had to be managed immediately to prevent any future complications like infection, corneal abrasion, blindnessetc.

A single dose of tetanus toxoid injection was given to the patient. The patient was taken up under general anesthesia for the procedure with aseptic precautions. Right transconjuctival incision was placed. The eye was retracted medially. Using fine mosquito forceps, the wooden foreign body was rotated to loosen it from the bony wall and taken out gently from the eye. Blunt dissection was then performed to remove the bony spicules that were holding the foreign body, from the lateral orbital wall. No major bleeding was encountered during the procedure .An infant nasogastric tube was inserted through the track of the foreign body for antibiotic wash. The wooden stick was found to be of $8.5 \mathrm{~cm}$ long. The eye was then washed with normal saline to flush out any dust and dirt. The wound closed with 7-0 vicryl.

Systemic and topical antibiotic as well as analgesics were prescribed for the patient. Also an eye patch was placed over the right eye. The patient was instructed to flush the eye with cold water frequently for a week and not to rub the eye. Also was instructed to perform aggressive mouth opening exercises.

The patient was recalled after 1 week for follow-up. A plain sagittal CT of the right orbit was taken after the removal of the foreign body to ensure that no pieces were left behind. The CT orbit showed no traces of the foreign body in the right orbit.

The patient has been on regular follow-up for 1 year with no complications.

\section{Discussion :}

Foreign bodies of the eye or orbit account for a small but significant part of ocular trauma. The significance of this type of injury is heightened by its association with occupation and the serious complications it causes both earlyand late. ${ }^{1}$

Penetrating ocular trauma is a leading cause of unilateral blindness. Different modes and settings of the trauma may necessitate different approaches to the management of such cases. ${ }^{2}$

The impacted foreign body in the orbit may be organic or inert. Organic foreign bodies like wood in our case need to be removed as soon as possible due to the associated high risk of infection especially if the patient is immunocompromised. ${ }^{2}$ Wood, with its porous consistency and organic nature, provides a good medium for microbial agents. Infection resulting from retained intraorbital wooden foreign bodies may lead to complications such as panophthalmitis, abscess, and fistula ${ }^{3}$. Inert materials like glass, plastic or steel are associated with lesser risk of infection and a decision to remove them should be based on factors like site of impingement, size of foreign body, potential of secondary injuries and hematoma. The physical characteristics of the foreign body like mass and shape are also of prognostic importance. Woodcock et al ${ }^{2}$ from UK had found that foreign bodies of greater mass were associated with worse visual outcome. Lid laceration and adnexal injuries have been found to be associated with the eventual enucleation of injured eye. 


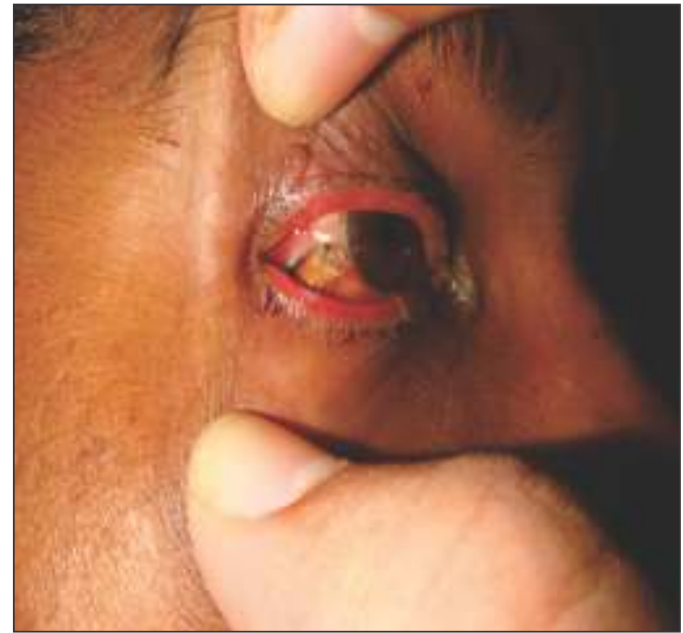

Fig 1 : Lateral profile of the patient showing $1 \mathrm{~cm}$ broom splinter in the right eye.

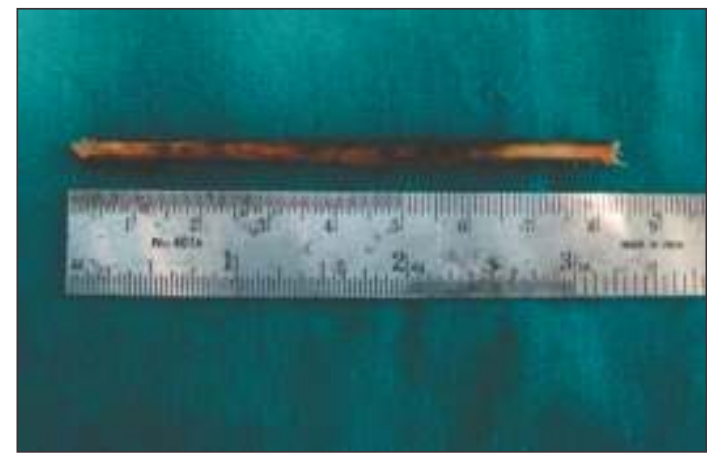

Fig 3 : The removed foreign body

Assessment through radiological images assists in the proper localization of the foreign body, estimation of its consistency and size, and evaluation of the response of surrounding orbital tissue. Additionally, it is useful in determining the integrity of the globe. The choice of imaging modality chiefly depends on the nature of the suspected foreign body. Plain film radiography is useful to localize radiopaque objects. However, plain films lack the capacity to demonstrate the object details, their exact location in relation to surrounding structures and tissue response or damage. Standardized ophthalmic ultrasonography (combination of standard A-mode and Bmode scanning) has been recommended as the imaging modality of choice during initial evaluation. Nevertheless, this diagnostic method requires specific expertise and technology that may not be available in many institutions. CT scanning has therefore been recommended as the imaging modality of choice in this situation. Ideally, a CT

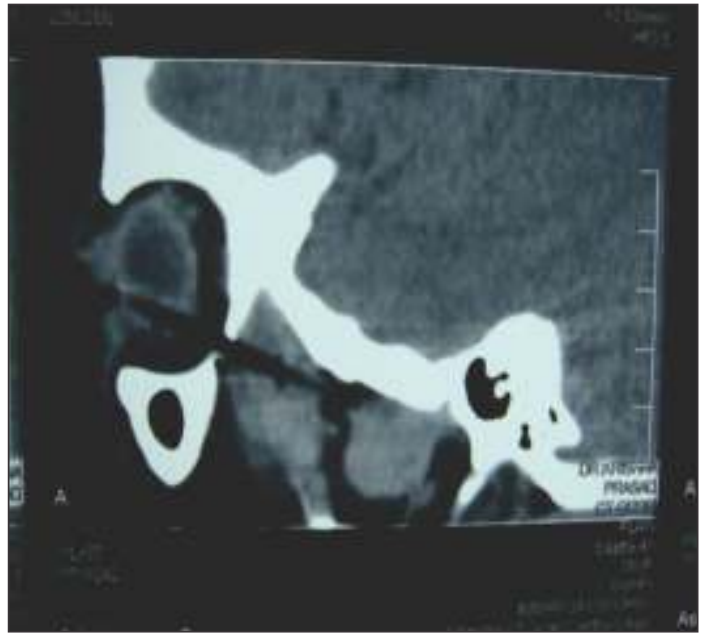

Fig 2 : Plain sagittal CT of the orbit

scan of the orbit must be ordered. Thin axial and coronal views of 1.0-1.5 mm cuts of the orbit are extremely useful to delineate the shape and for determining the composition of the foreign body. However, despite being highly sensitive and specific for detection of foreign bodies, CT scans may produce false negative results, particularly if the size of the foreign body is less than $0.5 \mathrm{~mm}$, and especially in the case of wooden objects. These are better seen with magnetic resonance imaging (M RI). However, an $M R I$ is contraindicated if the suspected foreign body is ferromagnetic. ${ }^{4}$

Retained organic foreign bodies are not often immediately apparent on imaging studies, making prompt removal difficult. Previous studies report that wood, depending on its level of hydration, can be isodense to either air or orbital fat on CT scan. ${ }^{5}$

Although the radiological appearance may show a great variety, $\mathrm{CT}$ imaging is the basic diagnostic technique. M RI is the method of second choice. ${ }^{6}$

Once an intraorbital foreign body is diagnosed, appropriate management includes culture of the wound (or foreign body if removed) and administration of antibiotic(s). Tetanus toxoid has to be administered according to the vaccination status.

The management and prognosis depend on the composition and location of the foreign body as well as the 
possible presence of secondary infection. ${ }^{7}$

Most metallic foreign bodies remain quiescent for a long period of time without causing any problems. So the general recommendation is to leave them alone in the absence of specific indications for removal.

However, organic foreign bodies like wood have a much higher incidence of potentially sight-threatening and lifethreatening complications. They may remain dormant for a variable period of time and manifest with delayed-onset orbital granuloma, cellulitis, abscess or chronic draining sinus. $^{8}$

Hence, surgical removal of organic intraorbital foreign bodies is recommended.

Large intraorbital foreign bodies usually slide between the eyeball and the orbital walls, but rarely cause severe trauma to the eye. However, immediate direct trauma to important intraconal structures may even damage the optic nerve. Paresis of the extraocular muscles and anaesthetic immobile globe has been reported. Rarely do the foreign bodies traverse the orbit into the cranium or to adjacent paranasal sinuses causing extensive damage to surrounding structures. ${ }^{9}$

In general, surgery to remove the foreign body is planned on the basis of the size and nature of the foreign body (organic objects are usually poorly tolerated), the location (anterior or posterior orbit) and the presence of other

\section{References:}

1. Khan M D, Kundi N, Zia M ohammed, Nazeer A F. A 61/2-years survey of intraocular and intraorbital foreign bodies in the North-west Frontier Province, Pakistan. British Journal of Ophthalmology, 1987:71:716-19.

2. Bhaduri G, Chattopadhyay S,Ghosh R P , Saurabh K, Goyal M. An unusual case of penetrating ocular trauma with metallic spoon. Indian Journal of Ophthalmology, 2010:58: 330-31.

3. Ho VT, M cGuckin JF Jr, Smergel EM . Intraorbital wooden foreign body: CT and M R appearance. AJNR AmJ Neuroradiol 1996:17:134-136.

4. Al-Mujaini A, Al-Senawi R, Ganesh A, Al-Zuhaibi S, Al-Dhuhli H. Intraorbital foreign body: clinical presentation, radiological appearance and management. Sultan Qaboos Univ. M ed J 2008:8:6974. injuries or foreign body-related complications (such as optic nerve compression, infections and extra ocular muscle involvement). ${ }^{5}$

Foreign body injuries in the orbital region can be approached with a combination of clinical suspicion, basic knowledge and diagnostic tests. The skill and experience of the surgeon are fundamental to decreasing the risk of iatrogenic injuries. ${ }^{?}$

We efficiently managed the case of wooden foreign body in the right orbit by removing the foreign body under general anesthesia and administering antibiotics and analgesic.

To conclude, intraocular foreign bodies remain a diagnostic dilemma and challenging experience to the maxillofacial surgeons. We have described an unusual case report of an intraorbital foreign body and the treatment was planned with accurate and detailed history as to the mechanism of injury, meticulous examination, as well as a CT scan of the orbit, which is the imaging modality of choice for detection and localization of the foreign body. The final outcome and visual prognosis depend greatly upon early diagnosis and also on frequent follow up visits. Foreign body injuries in the orbital region can be treated with a combination of clinical suspicion, basic knowledge and diagnostic tests and call for surgical skill and experience to decrease the risk of iatrogenic injury in relation to the inherent risk of retaining an organic intra-orbital foreign body.

5. Kim U R, Sivaraman KR. Penetrating orbital injuries from plant material during pond and river diving. Indian J Ophthalmol. 2013:61(2): 76-7.

6. Krimmel M, Cornelius CP, Stojadinovic S, Hoffmann J, Reinert S. Wooden foreign bodies in facial injury: a radiological pitfall. Int. J Oral M axillofac Surg. 2001: 30(5):445-7.

7. Moretti A, Laus M, Crescenzi D, Croce A. Peri-orbital foreign body: a case report. Journal of Medical Case Reports 2012, 6:91. doi: 10.1186/1752-1947-6-91.

8. John SS, Rehman TA, John D, Raju RS. M issed diagnosis of a wooden intra-orbital foreign body. Indian J Ophthalmol. 2008 Jul-Aug; 56(4): 322-4.

9. Agarwal PK, Kumar H, Srivastava PK.Unusual orbital foreign bodies. Indian J Ophthalmol. 19930ct; 41 (3):125-7. 